\title{
Lineshape Engineering in an All-Pass Ring Resonator with Backreflection Coupled to a Symmetrical Fabry-Perot Resonator
}

\author{
Vasily A. Melnikov", Iman S. Roqan \\ King Abdullah University of Science and Technology (KAUST), Physical Sciences and Engineering Division, Thuwal 23955-6900, Saudi Arabia \\ *Email: vasily.melnikov@kaust.edu.sa
}

\begin{abstract}
We derive transfer functions for an all-pass ring resonator with internal backreflection coupled to a symmetrical Fabry-Perot resonator and demonstrate electromagnetically induced transparency-like and Fano-like lineshapes tunable by backreflection in the ring resonator.

OCIS codes: (230.5750) Resonators; (130.0130) Integrated optics.
\end{abstract}

\section{Introduction}

Integrated ring resonators (RR) have numerous applications in active and passive photonic integrated circuits for light generation, detection, optical signal processing, and sensing [1,2], which require precise control on the position and lineshape of resonances. While in many cases, perfect Lorentz lineshape is desired some applications demand more complex resonance lineshapes. For example, narrow electromagnetically induced transparency (EIT) like resonances in RR-based devices can be used for light storage [3], while Fano-like resonances with steep edges and an improved extinction ratio are shown to increase the sensitivity of RR sensors [4] and reduce power threshold for optical switching on a chip [5]. In this work, we propose an all-pass RR with internal backreflection coupled to a symmetrical Fabry-Perot resonator (FPR) for resonance lineshape engineering and control. We derive the transfer functions for this system and demonstrate the opening of ultra-narrow EIT-like single and splitted resonances with quality factors more than 35 times higher than for individual RR or FPR. For these resonances, we show the possibility to tune single resonance lineshape from Lorentz to Fano-like type and to control the degree of splitting for splitted resonance by tuning the internal backreflection in RR.

\section{Theory}

First, by using space-domain coupled-mode theory (CMT) steady-state loop equations [6] we derived amplitude transfer functions for an all-pass RR with backreflection, as shown in Fig. 1a, for transmission in through port $T_{t l}$ and reflection in input port $T_{t 2}$ :

$$
\begin{aligned}
& T_{t 1}=\frac{S_{t 1}}{S_{0}}=\frac{C \exp (-i \varphi)-t}{t \cdot C \exp (-i \varphi)-1} \\
& T_{t 2}=\frac{S_{t 2}}{S_{0}}=\frac{i r \kappa^{2} \exp (-2 i \theta)}{\left(t \cdot t_{r} \exp (-i \varphi)-1\right)(t \cdot C \exp (-i \varphi)-1)} \\
& C=\frac{t_{r}-t \exp (-i \varphi)}{1-t \cdot t_{r} \exp (-i \varphi)}, \quad \theta=\varphi \cdot p, \quad p \in[0,1]
\end{aligned}
$$

where $t$ and $\kappa$ are the amplitude self-coupling and cross-coupling coefficients between access waveguide and RR, respectively; and parameter $\varphi=L_{R} \cdot\left[\left(2 \pi n_{\text {eff }} / \lambda\right)-(i \alpha / 2)\right]$ includes single-pass phase shift and propagation losses defined by the RR length $L_{R}=2 \pi R$, effective index $n_{\text {eff }}$, and attenuation coefficient $\alpha$.
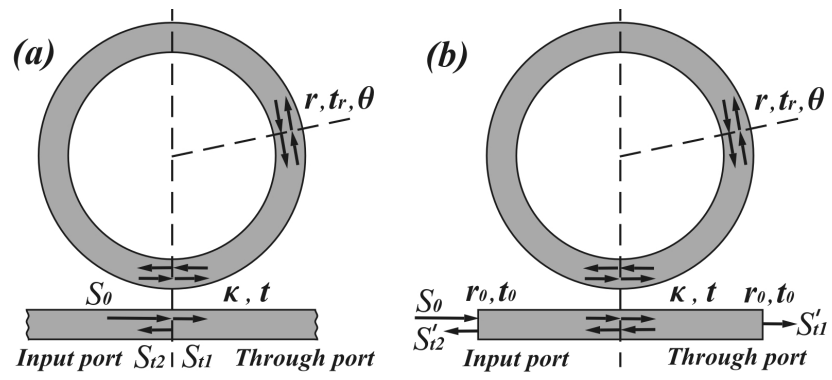

Fig. 1. Schematic of (a) all-pass RR with backreflection and $(b)$ the same RR coupled to FPR. 
The internal backreflection in the RR can be caused by locally introduced reflective element, such as slit, notch, bump or coupled particle, or it can be due to distributed modulation of the effective refractive index of the waveguide, e.g., grating or waveguide surface roughness. While the physical origin of the backreflection can be different, here it is lumped at a single reference plane at the phase position $\theta$ and has amplitude self-coupling and cross-coupling coefficients $t_{r}$ and $r$, respectively.

Second, we derived the amplitude transfer functions for an all-pass RR with backreflection coupled to a symmetrical FPR (Fig. 1b):

$$
\begin{aligned}
& T_{t 1}^{\prime}=\frac{S_{t 1}^{\prime}}{S_{0}}=\frac{\left(1-r_{0}^{2}\right) T_{t 1} \exp \left(-i \varphi_{0}\right)}{\left.1-r_{0}\left(T_{t 2}+T_{t 2}^{\prime}\right) \exp \left(-i \varphi_{0}\right)-r_{0}^{2}\left(T_{t 1}^{2}-T_{t 2} T_{t 2}^{\prime}\right) \exp \left(-2 i \varphi_{0}\right)\right)} \\
& T_{t 2}^{\prime}=\frac{S_{t 2}^{\prime}}{S_{0}}=\frac{\left.-r_{0}+\left(T_{t 2}+r_{0}^{2} T_{t 2}^{\prime}\right) \exp \left(-i \varphi_{0}\right)+r_{0}\left(T_{t 1}^{2}-T_{t 2} T_{t 2}^{\prime}\right) \exp \left(-2 i \varphi_{0}\right)\right)}{\left.1-r_{0}\left(T_{t 2}+T_{t 2}^{\prime}\right) \exp \left(-i \varphi_{0}\right)-r_{0}^{2}\left(T_{t 1}^{2}-T_{t 2} t_{t 2}^{\prime 2}\right) \exp \left(-2 i \varphi_{0}\right)\right)} \\
& T_{t 2}^{\prime}(\theta)=T_{t 2}(\theta=\varphi(p-1))
\end{aligned}
$$

where $T_{t 1}$ and $T_{t 2}$ are the transfer functions of a single all-pass RR with backreflection given by Eq. (1); $r_{0}$ is the amplitude reflection coefficient of the FPR mirrors (e.g., access waveguide facets); and parameter $\varphi_{0}=L_{0} \cdot\left[\left(2 \pi n_{\text {effo }} / \lambda\right)-\left(i \alpha_{0} / 2\right)\right]$ includes single-pass phase shift and propagation losses defined by FPR length $L_{0}$, its effective index $n_{\text {effo }}$, and attenuation coefficient $\alpha_{0}$. High values of $r_{0}$ can be achieved by using gratings or onedimensional photonic crystals fabricated in an access waveguide [7]. In contrast to equations obtained from timedomain CMT, these equations can be used to simulate all resonance lines simultaneously taking into account material and waveguide dispersion as well as dispersion of coupling coefficient and propagation losses.

\section{Results and discussion}

As a realistic system for numerical modeling we considered an all-pass RR with radius $R=10 \mu \mathrm{m}$ formed by a single-mode silicon photonic wire (SPW) [2] with effective index $n_{e f f}=2.15$ at a resonance wavelength $\lambda_{r}=1552.74$ $\mathrm{nm}$ coupled to FPR $\left(n_{\text {eff }}=n_{\text {effo }}=2.15, r_{0}=0.9\right)$ with coupling coefficient $\kappa=0.2$. In the calculations, we neglected dispersion of the effective index and attenuation and coupling coefficients in the spectral range of interest. Figure 2 presents the transmission spectra of single RR and FPR together with the spectra of the coupled RR+FPR system calculated using Eqs. (1-2) at $r=0$ for $L_{0}=L_{R} / 2$ (Figs. 2(a-c)) and $L_{0}=L_{R} / 2+\lambda_{r} /\left(4 n_{\text {eff }}\right.$ ) (Figs. 2(d-f)). Figures 2(a,d) are calculated for $\alpha=\alpha_{0} \approx 6.5 \mathrm{~cm}^{-1}$ corresponding to a critically coupled RR. Figures 2(b,e) magnified in Figures 2(c,f) are calculated with the assumption of negligible propagation losses.

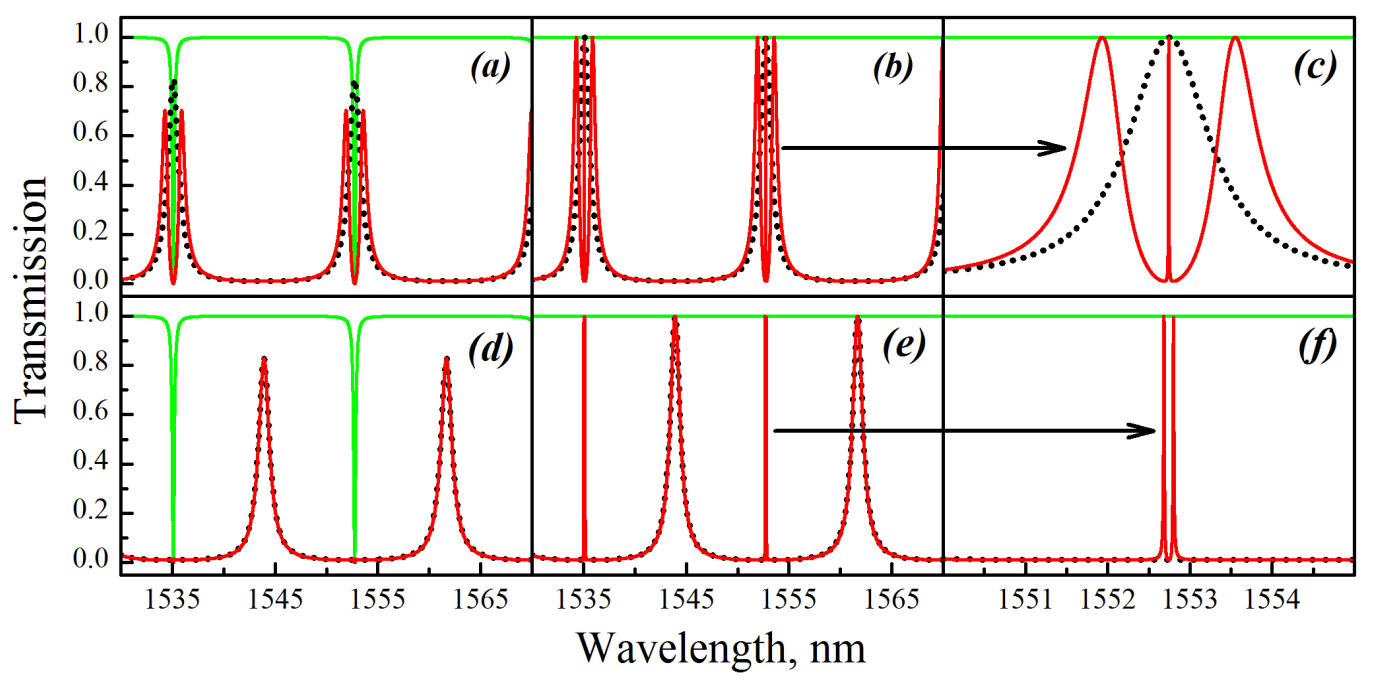

Fig. 2. Calculated transmission through an all-pass RR (green line), a FPR (black dotted line), and RR $+\mathrm{FPR}\left(\right.$ red line) for $(a-c) L_{0}=L_{R} / 2$ and $(d-f) L_{0}=L_{R} / 2+\lambda_{r} /\left(4 n_{e f f}\right)$.

When round-trip optical path lengths (OPLs) of RR and FPR are equal or differ by an integer number of wavelengths, the transmission of critically coupled resonators is reminiscent to a single FPR transmission with splitted resonances (Fig. 2a). However, if propagation losses are negligible in both RR and FPR, a narrow EIT-like resonance appears at the resonance wavelength of isolated resonators in surrounding of the splitted FPR resonance (Fig. 2c). The quality factor of this narrow resonance is $Q \sim 2.6 \times 10^{5}$ while a single FPR has $Q \sim 1300$ and a 
critically-coupled single $\mathrm{RR}$ has $Q \sim 6700$. This example indicates a generic approach for engineering narrow high$Q$ resonances by matching the OPLs of coupled resonators with moderate quality factors. Introduction of backreflection in RR leads to the red shift of the narrow resonance and modification of its shape from the symmetrical Lorentz to a completely asymmetric Fano-like line with a steep edge beneficial for low-threshold optical switching and sensing (Fig. 3a).

When round-trip OPLs of RR and FPR differ by an odd number of half-wavelengths, the resulting transmission of the critically coupled RR+FPR is equal to the transmission of a single FPR (Fig. 2d). For lossless resonators, two additional narrow resonances (Fig. 2f) with $Q \sim 1.3 \times 10^{5}$ (i.e., about half of $Q$ for the narrow resonance in Fig. 2c) appear close to the resonance wavelengths of a single RR visible in Fig. 2d. The splitting between these lines can be controlled by the changing backreflection coefficient of the RR without affecting the broad FPR resonances (Fig. 3b).

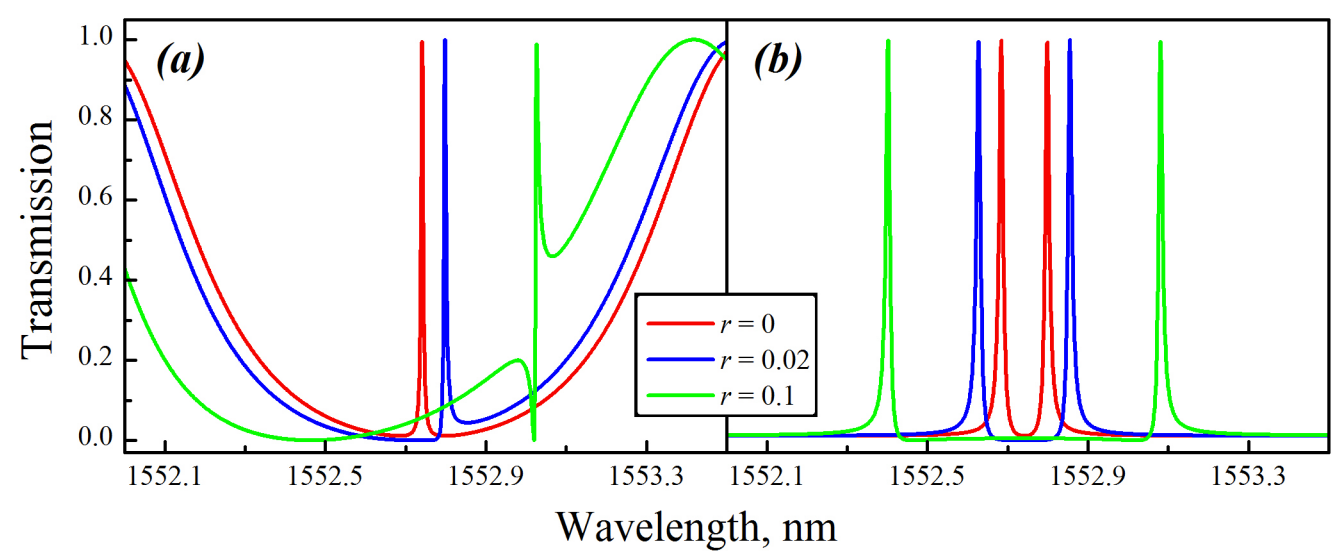

Fig. 3. Transmission spectra through RR+FPR with $(a) L_{0}=L_{R} / 2$ and (b) $L_{0}=L_{R} / 2+\lambda_{r} /\left(4 n_{e f f}\right)$ calculated for different values of amplitude backreflection coefficient $r$ in RR.

In both cases shown in Figures 2(c,f), the linewidth of EIT-like single and splitted resonances can be decreased by using RR of larger size with the OPL equal to an integer multiple of the FPR OPL. For the splitted resonance (Fig. 2f) this also brings the two lines closer to each other. It should be noted that all these narrow resonances become obscured or completely vanish (Figs. 2(a,d)) for significant losses in the RR in agreement with Ref. 8 .

\section{Conclusion}

We derive universal analytical equations for the transfer functions of a RR with backreflection coupled to a symmetrical FPR and demonstrate, on example of SPW based resonators, formation of ultra-narrow EIT-like single, splitted and Fano-like resonances tunable by the backreflection coefficient of the RR. For the particular case, quality factors of these resonances $\left(Q \sim 10^{5}\right)$ significantly exceed the moderate quality factors of individual RR and FPR $\left(Q \sim 10^{3}\right)$. The opening of high- $Q$ resonances with controllable lineshape can be used for a number of applications, including lasing, frequency conversion, pulse shaping and generation, light storage and delay, optical switching, modulation, signal filtering, sensing, and metrology.

\section{References}

[1] J. Heebner, R. Grover, and T. Ibrahim, Optical microresonators: theory, fabrication and applications, 1st ed., Springer Series in Optical (Sciences, Springer: London, 2008).

[2] W. Bogaerts, P. De Heyn, T. Van Vaerenbergh, K. De Vos, S. Selvaraja, T. Claes, P. Dumon, P. Bienstman, D. Van Thourhout, R. Baets, "Silicon microring resonators," Laser \& Phot. Rev. 6, 47-73 (2012).

[3] Q. Xu, P. Dong, and M. Lipson, “Breaking the delay-bandwidth limit in a photonic structure,” Nature Phys. 3, $406-410$ (2007).

[4] H. Yi, D. S. Citrin, and Z. Zhou, "Highly sensitive silicon microring sensor with sharp asymmetrical resonance," Opt. Express 18, 2967-2972 (2010).

[5] L. Y. Mario, S. Darmawan, and M. K. Chin, "Asymmetric Fano resonance and bistability for high extinction ratio, large modulation depth, and low power switching," Opt. Express 14, 12770-12781 (2006).

[6] B. Little, J. Laine, and S. Chu, "Surface-roughness-induced contradirectional coupling in ring and disk resonators," Opt. Lett. 22, 4-6 (1997).

[7] M. W. Pruessner, T. H. Stievater, and W. S. Rabinovich, "Integrated waveguide Fabry-Perot microcavities with silicon/air Bragg mirrors," Opt. Lett. 32, 533-535 (2007).

[8] D. D. Smith, H. Chang, K. A. Fuller, A. T. Rosenberger, and R. W. Boyd, “Coupled-resonator-induced transparency,” Phys. Rev. A 69, 063804(1-6) (2004). 